Fernanda Pititone RodriGues ${ }^{1}$

SILVIO MARTINELLI ${ }^{1}$

RoBerto EDUARDO BitTAR ${ }^{2}$

Rossana Pulcineu VierRa Francisco 2

MARCELO ZUGABB ${ }^{2}$

Artigo Original

Palavras-chave

Retardo do crescimento fetal Recém-nascido pequeno para idade gestacional

Peso ao nascer Diagnóstico pré-natal

Keywords

Fetal growth retardation Infant, small for gestational age

Birth weight

Prenatal diagnosis

\title{
Comparação entre duas curvas de crescimento para o diagnóstico de recém-nascidos pequenos para a idade gestacional
}

\author{
Comparison between two growth curves for small for \\ gestational age diagnosis
}

\section{Resumo}

OBJETIVO: Foi comparar a aplicação de duas curvas de crescimento para o diagnóstico de recém-nascidos pequenos para a idade gestacional (PIG), utilizando o percentil 10 como referência. MÉTODOS: Estudo retrospectivo com informações do parto de 20.567 recém-nascidos vivos, de gestações únicas, ocorridos entre janeiro de 2003 e junho de 2014, divididos em grupos por idade gestacional: (a) 23 a 26, (b) 26 a 29, (c) 29 a 32 , (d) 32 a 35, (e) 35 a 38, (f) 38 a 41 e (g) $>41$ semanas. Os dados foram pareados e os grupos comparados por teste de igualdade de proporções segundo método de McNemar. $\bigcirc$ nível de significância foi estabelecido em $p<0,05$. RESULTADOS: A curva de Alexander apresentou maior taxa de diagnóstico de PIG do que a curva de Fenton em todas as faixas de idade gestacional até a $41^{\circ}$ semana, com maior diferença entre as curvas entre 32 e 35 semanas (18,5\%). No período entre 37 e 40 semanas, o diagnóstico de PIG, empregando-se a curva de Alexander, superou o de Fenton em 9, $1 \%$ dos casos. Com exceção dos grupos entre 23 e 26 semanas, todas as outras faixas de idade gestacional mostraram-se significativamente diferentes quanto ao diagnóstico de RN PIG. CONCLUSÃO: A curva de Fenton é um instrumento estatístico mais robusto, construída com informações mais recentes, e permite a avaliação do crescimento por três parâmetros e por sexo.

\section{Abstract}

PURPOSE: It was to compare the use of two growth curves for the diagnosis of small-for-gestational-age (SGA) infants, having the $10^{\text {th }}$ percentile as reference. METHODS: In a retrospective study, data of 20,567 singleton live births from January 2003 to June 2014 were analyzed, and divided according to gestational age: (a) 23 to 26, (b) 26 to 29, (c) 29 to 32 , (d) 32 to 35, (e) 35 to 38, (f) 38 to 41 and (g) >41 weeks. Data were paired and analyzed using the McNemar test, with the level of significance set at 0.05. RESULTS: The curve designed by Alexander indicated a higher percentage of diagnosis of SGA than the curve constructed by Fenton for every category of gestational age up to 41 weeks, more markedly in the 32-35 week group (18.5\%). Between 37 and 40 weeks of gestational age, Alexander's curve exceeded Fenton's curve in $9.1 \%$ of the cases in the diagnosis of SGA. CONCLUSIONS: The Fenton curve provides a more accurate evaluation of an infant's growth since it is gender-specific and allows measurement of three parameters. It has also been constructed with newer data and more sophisticated statistical tools.

Correspondência

Fernanda Pipitone Rodrigues Avenida Doutor Eneas de Carvalho Aguiar, 255 - Cerqueira César (EP: 05403-010 São Paulo (SP), Brasil

Recebido $06 / 10 / 2014$

Aceito com modificacões

$17 / 11 / 2014$
Departamento de Obstetrícia e Ginecologia do Hospital das Clínicas da Faculdade de Medicina da Universidade de São Paulo USP - São Paulo (SP), Brasil.

Hospital das Clínicas, Faculdade de Medicina, Universidade de São Paulo - USP - São Paulo (SP), Brasil.

2Departamento de Obstetrícia e Ginecologia, Faculdade de Medicina, Universidade de São Paulo - USP - São Paulo (SP), Brasil.

Conflitos de interesses: não há 


\section{Introdução}

A restrição do crescimento fetal (RCF) é assunto de grande relevância por ser uma das principais complicações da gravidez e acarretar elevados índices de morbimortalidade perinatais $^{1,2}$. Há relação direta entre baixo percentil de peso ao nascer e mau prognóstico perinatal, com os maiores riscos se apresentando em fetos com percentil menor do que $3^{3,4}$. Somado a isso, a RCF está associada a hipertensão arterial, dislipidemia, doença coronariana e diabetes mellitus na vida adulta $^{5-7}$. No entanto, não há, até o momento, tratamento clínico que previna ou corrija esse déficit. Tentar esclarecer a etiologia e definir o momento mais adequado para o parto é, portanto, um desafio na prática obstétrica ${ }^{8}$.

A RCF é habitualmente definida na literatura como peso fetal inferior ao percentil 10 para a idade gestacional, empregando-se, na maioria das vezes, curvas-padrão próprias de cada população ${ }^{9}$. Apesar de amplamente utilizada, essa definição tem sua limitação por não fazer distinção entre fetos pequenos constitucionais e fetos pequenos em razão de um processo patológico que os impediu de atingir seu potencial genético. Também não há consenso se a curva-padrão deve ser baseada no peso de nascimento, no peso fetal estimado pela ultrassonografia ou em curvas individualizadas (ajustadas por fatores maternos ${ }^{10-13}$. A crítica mais relevante em relação à utilização do peso de nascimento é o fato de recém-nascidos (RN) prematuros apresentarem também restrição de crescimento com maior frequência. A limitação da estimativa ultrassonográfica de peso é sua imprecisão, uma vez que estima os valores com o feto intraútero ${ }^{14}$.

O rastreamento pré-natal da RCF na população obstétrica envolve identificação de fatores de risco e avaliação indireta do crescimento fetal pelo exame físico. Medidas seriadas da altura uterina abaixo do percentil 10 para a idade gestacional elevam a suspeita de restrição e demandam investigação aprofundada ${ }^{15-17}$. Apesar da suspeita de RCF durante a gestação, esse diagnóstico só pode ser confirmado após o parto, quando definimos um recém-nascido pequeno para idade gestacional (PIG), cujo peso de nascimento é também inferior ao percentil 10 para a idade gestacional ${ }^{9}$.

Diversos estudos foram feitos sobre curvas de crescimento fetal, e diferentes padrões populacionais foram criados ao longo dos anos ${ }^{18,19}$. Battaglia e Lubchenco 9 utilizaram pesos de nascidos vivos em Denver, Estados Unidos da América, para definir o intervalo entre os percentis 10 e 90 como adequado para idade gestacional. Já Usher e McLean $^{20}$ escolheram critérios mais rígidos, adotando como RCF pesos inferiores ao percentil 3. Entre as várias curvas de crescimento fetal adotadas, destacam-se a curva de Alexander et al. ${ }^{21} \mathrm{e}$, mais recentemente, a de Fenton e $\mathrm{Kim}^{22}$, que serão revisadas neste estudo.

Em 1996, Alexander et al. ${ }^{21}$ desenvolveram curva de referência nacional norte-americana valendo-se do peso de nascimento de nascidos vivos em 1991 de gestações únicas de mãe residentes dos EUA. A curva de Alexander et al. ${ }^{21}$ foi amplamente utilizada como referência nacional para crescimento fetal nos EUA até 2010, quando foi substituída pela de Olsen et al..$^{23}$, uma nova referência americana. Em 2013, Fenton e Kim ${ }^{22}$ realizaram uma metanálise incluindo estudos de seis países desenvolvidos e abrangendo amostra importante de recém-nascidos com registros de peso, comprimento e perímetro cefálico. A curva se valeu ainda do padrão de crescimento da $\mathrm{OMS}^{24}$ por 10 semanas, permitindo um acompanhamento clínico até 50 semanas de idade gestacional corrigida. Esse cálculo é feito para ajuste da idade cronológica em função do grau de prematuridade. Considerando-se ideal nascer com 40 semanas de idade gestacional, subtrai-se da idade cronológica as semanas que faltam para atingir 40 semanas $^{24}$.

Este estudo teve por objetivo comparar a aplicação de duas importantes curvas de crescimento fetal, Alexander et al. ${ }^{21}$ e Fenton e Kim ${ }^{22}$, para o diagnóstico de RN PIG, utilizando o percentil 10 como referência.

\section{Métodos}

Trata-se de estudo retrospectivo com dados de parto de 20.567 RN vivos, de gestações únicas, na Clínica Obstétrica do Hospital das Clínicas da Faculdade de Medicina da Universidade de São Paulo (HCFMUSP), entre janeiro de 2003 e junho de 2014. Esses dados foram selecionados do Programa da Enfermaria da Clínica Obstétrica (Microsoft Access 2007).

O presente estudo não recebeu suporte financeiro ou equipamentos ou medicamentos de nenhuma outra instituição, e não apresenta conflitos de interesse.

Os recém-nascidos foram divididos por grupos conforme a idade gestacional:

- 23 a 25 semanas e 6 dias $(n=185)$;

- 26 a 28 semanas e 6 dias $(n=421)$;

- 29 a 31 semanas e 6 dias $(n=670)$;

- 32 a 34 semanas e 6 dias $(n=1.421)$;

- $\quad 35$ a 37 semanas e 6 dias $(n=6.725)$;

- 38 a 40 semanas e 6 dias $(n=10.562)$; e

- 41 semanas ou mais $(n=583)$.

Os grupos foram comparados quanto ao diagnóstico de PIG (peso de nascimento abaixo do percentil 10) segundo as curvas de Alexander et al. ${ }^{21}$ e Fenton e Kim ${ }^{22}$, utilizando software próprio do Setor de Baixo Peso Fetal (Microsoft Access 2007).

As seguintes variáveis foram analisadas: idade gestacional no momento do parto; percentil de peso para a idade gestacional segundo Alexander et al. ${ }^{21}$; percentil de peso para a idade gestacional segundo Fenton e $\mathrm{Kim}^{22}$ e número de recém-nascidos com peso inferior ao percentil 10 em cada grupo 
Após compilados, os dados foram pareados, e os grupos comparados por teste de igualdade de proporções segundo método de McNemar. O nível de significância foi estabelecido em $\mathrm{p}<0,05$.

\section{Resultados}

A Tabela 1 mostra os recém-nascidos com peso abaixo do percentil 10, segundo as curvas de Alexander et al. ${ }^{21}$ e Fenton e $\mathrm{Kim}^{22}$, divididos pelos grupos de idade gestacional.

Quando se aplica a curva de Alexander et al. ${ }^{21}$, detecta-se maior porcentagem de RN PIG em todas as faixas, com exceção do grupo de idade gestacional acima de 41 semanas. Essa diferença foi máxima entre 32 e 35 semanas $(18,5 \%)$.

Também foi avaliado, em razão de sua importância na prática clínica, o período entre a $37^{\mathrm{a}}$ e a $40^{\mathrm{a}}$ semana. Nessa faixa, a curva de Alexander et al. ${ }^{21}$ superou a de Fenton e $\mathrm{Kim}^{22}$ em 9,1\%.

Com exceção dos grupos entre 23 e 26 semanas, todas as outras faixas de idade gestacional mostraram-se significativamente diferentes quanto ao diagnóstico de RN PIG, como mostra a Tabela 2.

Tabela 1. Porcentagem de recém-nascidos abaixo do percentil 10 segundo a curva de Alexander et al. ${ }^{21}$ e Fenton e Kimm ${ }^{22}$ por faixa de idade gestacional, na amostra de recémnascidos vivos nascidos entre janeiro de 2003 e junho de 2014

\begin{tabular}{|c|c|c|c|}
\hline IG & $\begin{array}{c}\text { Alexander } \\
<\mathrm{p} 10\end{array}$ & $\begin{array}{c}\text { Fenton } \\
<p 10\end{array}$ & $\begin{array}{c}\text { Diferença } \\
\quad \%\end{array}$ \\
\hline $23-26$ & 17,8 & 16,8 & 1,0 \\
\hline $26-29$ & 35,2 & 28,5 & 6,7 \\
\hline 29-32 & 41,5 & 23,6 & 17,9 \\
\hline $32-35$ & 44,1 & 25,6 & 18,5 \\
\hline $35-38$ & 35,2 & 20,0 & 15,2 \\
\hline 38-41 & 18,8 & 14,2 & 4,6 \\
\hline$>41$ & 10,1 & 27,3 & $-17,2$ \\
\hline $37-40$ & 24,3 & 15,2 & 9,1 \\
\hline
\end{tabular}

IG: idade gestacional

Tabela 2. Aplicação do teste de McNemar por grupos, conforme a idade gestacional, para comparação da taxa de recém-nascidos pequenos para a idade gestacional segundo curva de Alexander et al. ${ }^{21}$ e Fenton e Kim ${ }^{22}$, com dados de recém-nascidos vivos entre janeiro de 2003 e junho de 2014

\begin{tabular}{|c|c|c|c|}
\hline $\begin{array}{l}\text { Idade gestacional } \\
\text { (semanas) }\end{array}$ & Número de RN & Estatística $\left(\chi^{2}\right)$ & Valor $p$ \\
\hline $23-26$ & 185 & 0,7 & 0,4 \\
\hline $26-29$ & 421 & 28,0 & $<0,01$ \\
\hline 29-32 & 670 & 120,0 & $<0,01$ \\
\hline $32-35$ & 1.421 & 263,0 & $<0,01$ \\
\hline $35-38$ & 6.725 & 1021,0 & $<0,01$ \\
\hline $38-41$ & 10.562 & 350,9 & $<0,01$ \\
\hline Acima de 41 & 583 & 100,0 & $<0,01$ \\
\hline
\end{tabular}

RN: recém-nascido
Isso pode ser mais bem observado quando comparamos as duas curvas, avaliando só os casos com peso de nascimento abaixo do percentil 10 (Figuras 1 e 2). Especialmente após a $26^{a}$ semana, o percentil 10 da curva de Alexander et al. ${ }^{21}$ mostra valores mais elevados até próximo de 40 semanas. Essa diferença se mostra mais evidente quando se compara as duas curvas para meninas.

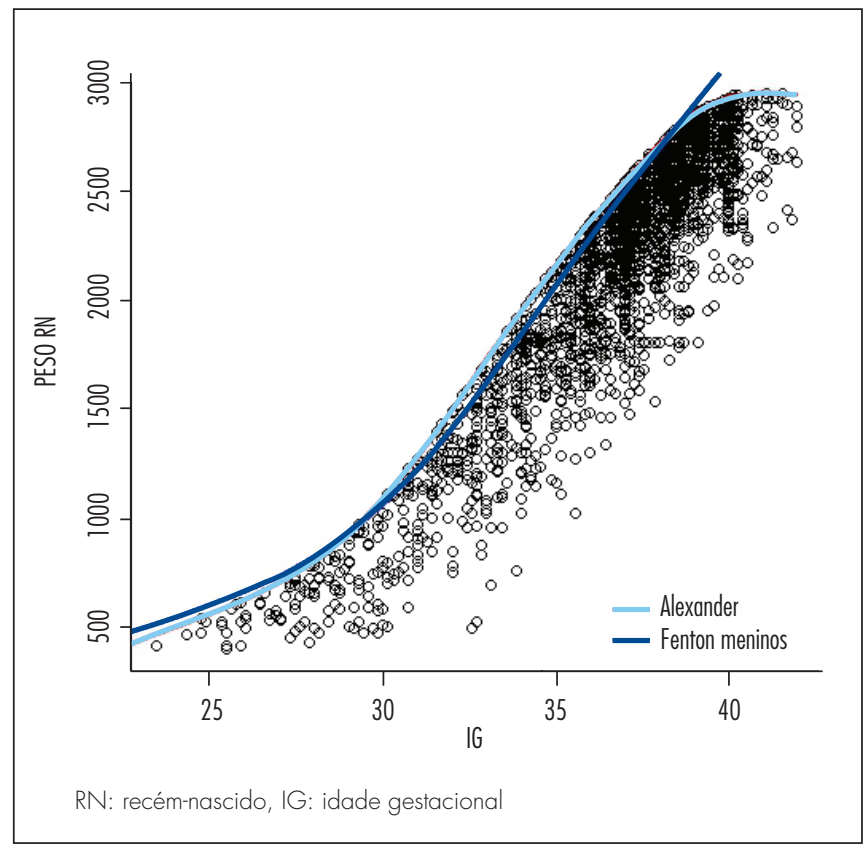

Figura 1. Pesos de recém-nascidos e percentil 10 segundo Alexander et al. ${ }^{21}$ e Fenton e $\mathrm{Kim}^{22}$ meninos

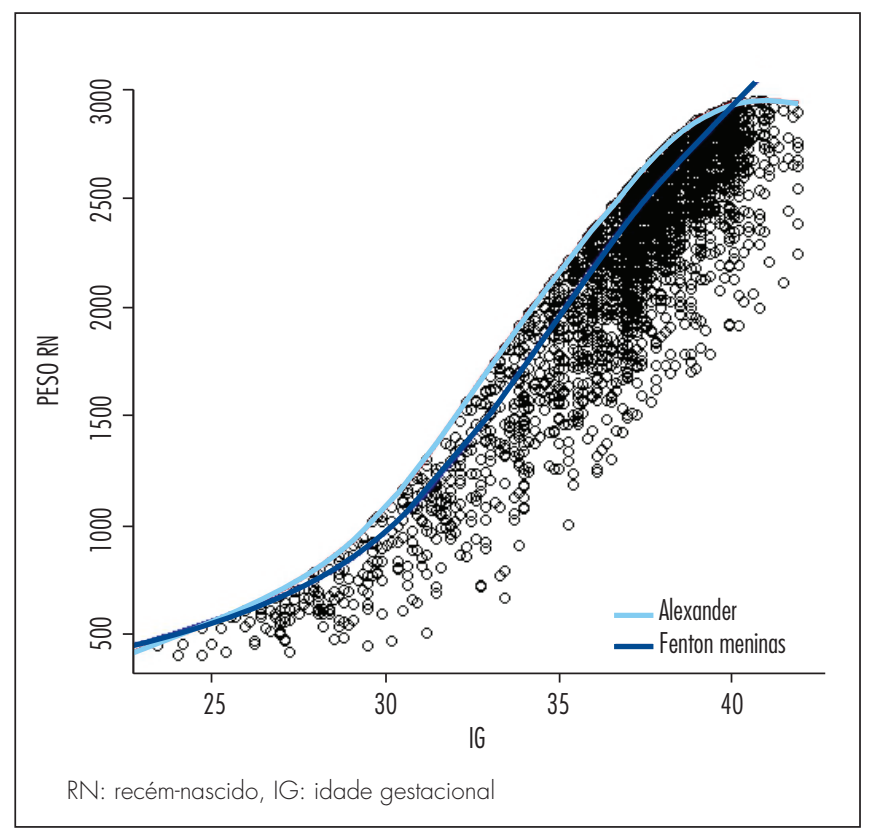

Figura 2. Pesos de recém-nascidos e percentil 10 segundo Alexander et al. ${ }^{21}$ e Fenton e $\mathrm{Kim}^{22}$ meninas 


\section{Discussão}

Embora tenha havido múltiplos estudos em busca de um referencial de crescimento fetal, ainda não há na literatura consenso sobre um padrão, ou seja, uma definição de normalidade. Também não encontramos estudos até o momento que tenham comparado o diagnóstico de PIG pelas Curvas de Alexander et al. ${ }^{21}$ e Fenton e $\mathrm{Kim}^{22}$.

Alexander et al. ${ }^{21}$ se propuseram a criar uma curva de referência de crescimento fetal norte-americana, comparar a curva obtida com trabalhos previamente publicados e estabelecer curvas específicas para cada sexo. O estudo tomou como amostra 3.134.879 nascidos vivos de gestações únicas de mães residentes americanas e considerou o percentil 10 como ponto de corte para PIG. O cálculo da idade gestacional foi baseado na data da última menstruação (DUM).

Alexander et al. ${ }^{21}$ selecionaram curvas de crescimento fetal de diferentes regiões e grupos étnicos nos EUA para comparar com seu estudo (Curvas de Lubchenco, Brenner, Williams e Ott). Entre os quatro estudos selecionados para comparação, o percentual de PIG variou de 2,7 a 15,6\%, estando Brenner e Lubchenco muito abaixo dos $10 \%$ esperados $(4,5$ e $2,7 \%$, respectivamente). Os autores discutem que as curvas publicadas anteriormente subestimaram o diagnóstico de RCF, com maior evidência entre a $33^{\mathrm{a}}$ e a $38^{\mathrm{a}}$ semana de idade gestacional, e que, portanto, esse diagnóstico varia enormemente conforme a curva utilizada. Por essa razão, consideram que muitas curvas publicadas já não representam material atualizado para referência de percentil 10 do peso de nascimento para a idade gestacional. Alexander et al. ${ }^{21}$ postulam razões para a disparidade entre sua curva e as anteriormente publicadas. Algumas foram construídas décadas antes e, portanto, refletem em parte uma melhora nutricional e de crescimento da população americana. Outras talvez não sejam representativas de toda população. A curva de Alexander et al. ${ }^{21}$, além de abranger amostra muito ampla, foi baseada em um banco de dados nacional e não em um único serviço ou região, o que permite ao estudo validação externa. Por outro lado, apesar de ser um instrumento útil para comparação, a curva de Alexander et al. ${ }^{21}$ não é adequada para estudo de grupos éticos específicos. Outra limitação do trabalho é o fato de utilizar a DUM para o cálculo da idade gestacional, o que pode reduzir a acurácia dos dados.

Fenton e $\mathrm{Kim}^{22}$ realizaram uma metanálise abrangendo estudos de seis países desenvolvidos (Alemanha, Austrália, Canadá, Escócia, EUA e Itália), produzidos entre 1987 e 2012, e incluíram amostra importante de recém-nascidos: 3.986 .456 com registros de peso, 151.527 de comprimento e 173.612 de perímetro cefálico. O estudo teve por objetivo revisar seu antecessor Fenton Preterm Growth Chart ${ }^{25}$ de 2003 , especificamente para: incluir dados mais recentes de peso ao nascer; harmonizar a curva de crescimento de pré-termos com a curva da Organização Mundial da Saúde ${ }^{24}$; suavizar a transição entre as duas curvas sem perder a integridade dos dados de 22 a 36 e com 50 semanas (pós-natal); produzir curvas específicas para cada sexo; e mudar a escala do eixo x dos gráficos para idades gestacionais precisas, a fim de melhorar a acurácia.

Os estudos incluídos na metanálise mostraram boa concordância, em especial no percentil 50 e seus inferiores. Quando analisados por idade gestacional, houve semelhança até 36 semanas e novamente com 50 semanas. Fenton e $\mathrm{Kim}^{22}$ concluem que o padrão de crescimento ideal ainda permanece indefinido. Discutem que utilizar o peso de recém-nascidos pré-termo é medida enviesada, já que há maior probabilidade de um prematuro apresentar restrição de crescimento, e que, portanto, fetos que permanecem no útero podem carregar diferenças importantes.

$\mathrm{Na}$ amostra deste estudo, a curva de Alexander et al. ${ }^{21}$ superou a de Fentone $\mathrm{Kim}^{22}$ no número de diagnósticos de PIG em todas as faixas de idade gestacional até 41 semanas, com maior diferença entre a $32^{\text {a }}$ e a $35^{\text {a }}$ semana $(18,5 \%)$.

Há implicação clínica na taxa de diagnóstico de PIG ao se utilizar Fenton e $\mathrm{Kim}^{22}$. Na faixa de idade gestacional entre 37 e 40 semanas, quando é indicada a resolução da grande maioria dos casos suspeitos de RCF, a diferença no diagnóstico foi de 9,1\%.

Concluímos que a curva de Fenton e Kim ${ }^{22}$ consiste em instrumento estatístico mais robusto, construída com informações mais recentes e que permite a avaliação do crescimento por três parâmetros e por sexo. $\mathrm{Na}$ interface com a Pediatria, não se pode deixar de citar outra vantagem da curva de Fenton e $\mathrm{Kim}^{22}$ : por ter harmonizado os dados com a curva da $\mathrm{OMS}^{24}$ a partir da $40^{a}$ semana, ela permite ao pediatra o acompanhamento do crescimento até 50 semanas de idade gestacional corrigida. A correção da idade cronológica em função da prematuridade é fundamental para o correto diagnóstico do desenvolvimento nos primeiros anos de vida e para diminuir o risco de classificar erroneamente prematuros como anormais. A utilização da curva de Fenton e $\mathrm{Kim}^{22}$ implica menor número de crianças que terão cuidados especiais no berçário, segundo esse critério, e, portanto, menores custos hospitalares. 
1. Boulet SL, Alexander GR, Salihu HM, Kirby RS, Carlo WA. Fetal growth risk curves: defining levels of fetal growth restriction by neonatal death risk. Am J Obstet Gynecol. 2006; 195(6):1571-7.

2. Gardosi J, Madurasinghe V, Williams M, Malik A, Francis A. Maternal and fetal risk factors for stillbirth: population based study. BM. 2013;346:f108.

3. Mclntire DD, Bloom SL, Casey BM, Leveno KJ. Birth weight in relation to morbidity and mortality among newborn infants. $\mathrm{N}$ Engl J Med. 1999;340(16):1234-8.

4. Pilliod RA, Cheng YW, Snowden JM, Doss AE, Caughey AB. The risk of intrauterine fetal death in the small-for-gestational-age fetus. Am J Obstet Gynecol. 2012;207(4):318.e 1-6.

5. Szostak-Wegierek D. Intrauterine nutrition: long-term consequences for vascular health. Int J Womens Health. 2014;6:647-56.

6. Longo S, Bollani L, Decembrino L, Di Comite A, Angelini M, Stronati $M$. Short-term and long-term sequelae in intrauterine growth retardation (IUGR). J Matern Fetal Neonatal Med. 2013;26(3):222-5.

7. Kanaka-Gantenbein C, Mastorakos G, Chrousos GP. Endocrinerelated causes and consequences of intrauterine growth retardation. Ann N Y Acad Sci. 2003;997:150-7.

8. Aviram A, Yogev Y, Bardin R, Meizner I, Wiznitzer A, Hadar E. Small for gestational age newborns - does pre-recognition make a difference in pregnancy outcome? J Matern Fetal Neonatal Med. 2014:1-5. [Epub ahead of print]

9. Battaglia FC, Lubchenco LO. A practical classification of newborn infants by weight and gestational age. J Pediatr. 1967;71 (2): 159-63.

10. Gardosi J, Chang A, Kalyan B, Sahota D, Symonds EM. Customised antenatal growth charts. Lancet. 1992;339(8788):283-7.

11. Zhang J, Mikolajczyk R, Grewal J, Neta G, Klebanoff M. Prenatal application of the individualized fetal growth reference. Am J Epidemiol. $2011 ; 173(5): 539-43$.

12. Carberry $A E$, Gordon A, Bond DM, Hyett J, Raynes-Greenow $\mathrm{CH}$, Jeffery HE. Customised versus population-based growth charts as a screening tool for detecting small for gestational age infants in low-risk pregnant women. Cochrane Database Syst Rev. 2014;5:CD008549.

13. Larkin JC, Hill LM, Speer PD, Simhan HN. Risk of morbid perinatal outcomes in small-for-gestational-age pregnancies: customized compared with conventional standards of fetal growth. Obstet Gynecol. 2012;119(1):21-7.

14. Liao P, Park AL, Berger H, Ray JG. Using estimated fetal weight from ultrasonography at 18 to 22 weeks to predict gestational diabetes mellitus and newborn macrosomia. J Obstet Gynaecol Can. 2014;36(8):688-91.

15. Martinelli S, Bittar RE, Zugaib M. [Prediction of fetal growth restriction by measurement of uterine height]. Rev Bras Ginecol Obstet. 2004;26(5):383-9. Portuguese.

16. Sparks TN, Cheng YW, McLaughlin B, Esakoff TF, Caughey AB. Fundal height: a useful screening tool for fetal growth? J Matern Fetal Neonatal Med. $2011 ; 24(5): 708-12$.

17. Roex A, Nikpoor P, van Eerd E, Hodyl N, Dekker G. Serial plotting on customised fundal height charts results in doubling of the antenatal detection of small for gestational age fetuses in nulliparous women. Aust N Z J Obstet Gynaecol. 2012;52(1):78-82.

18. Goldenberg RL, Cutter GR, Hoffman HJ, Foster JM, Nelson KG, Hauth JC. Intrauterine growth retardation: standards for diagnosis. Am J Obstet Gynecol. 1989;161 (2):271-7.

19. Riddle WR, DonLevy SC. Generating expected growth curves and Z-scores for premature infants. J Perinatol. 2010;30(1 1):741-50.

20. Usher R, McLean F. Intrauterine growth of live-born Caucasian infants at sea level: standards obtained from measurements in 7 dimensions of infants born between 25 and 44 weeks of gestation. J Pediatr. 1969;74(6):901-10.

21. Alexander GR, Himes JH, Kaufman RB, Mor J, Kogan M. A United States national reference for fetal growth. Obstet Ginecol. 1996;87(2):163-8.

22. Fenton TR, Kim JH. A systematic review and meta-analysis to revise the Fenton growth chart for preterm infants. BMC Pediatr. 2013;13:59.

23. Olsen IE, Groveman SA, Lawson ML, Clark RH, Zemel BS. New intrauterine growth curves based on United States data. Pediatrics. 2010;125(2):e214-24.

24. World Health Organization [Internet]. The WHO Child Growth Standards. Geneva: WHO; 2014 [cited 2014 Ago 20]. Available from: <http://www.who.int/childgrowth/standards/en/>

25. Fenton TR. A new growth chart for preterm babies: Babson and Benda's chart updated with recent data and a new format. BMC Pediatr. 2003;3:13. 